\title{
Low Computational Cost Model for Convective Heat Transfer from Submarine Cables
}

\author{
G. Callender, D.J. Ellis, K.F. Goddard, J.K. Dix, J.A. Pilgrim, Member, IEEE and M. Erdmann
}

\begin{abstract}
The ampacity of submarine cable circuits is strongly influenced by heat transfer in the marine environment surrounding the cable. It has been demonstrated in previous work that for high permeability sediments convective heat transfer can play a significant role using both bespoke two dimensional models and experimental investigations [1,2]. This paper introduces a one dimensional model which is capable of calculating cable temperatures within both convective and conductive sediments. Agreement between the one dimensional model and a two dimensional simulation was found to be within $1.5^{\circ} \mathrm{C}$. The model is used to demonstrate that the ampacity of power cables may be significantly increased due to convective heat transfer. Further, the one dimensional model offers significant savings in computational time and cost compared to the two dimensional equivalent model. This allows the analysis of large DTS data sets in order to calculate: dynamic ratings; burial depths; and the long-term (annual to decadal) performance of the cable.
\end{abstract}

Index Terms - submarine power cables, convective heat transfer, finite element simulation, thermal models

\section{INTRODUCTION}

$\mathrm{B}$ URIED submarine high voltage (HV) cables are increasingly utilized in modern electrical networks; important examples are grid interconnectors and offshore wind farm circuits [3, 4]. For buried cables on land the environment surrounding the cable can be modeled with relative ease and can be used in thermal resistance formulae to produce current ratings $[5,6]$. This is in stark contrast to the submarine environment where migratory bedforms can alter burial depth, and sediment properties can be highly inhomogeneous [7]. For many submarine cables this issue is complicated by the long length of routes, where the cable will be buried in a variety of sediment types with varying thermal properties [7,8].

Fiber optic cables are typically installed within submarine $\mathrm{HV}$ cables in order to provide condition monitoring. Of particular importance are distributed temperature sensing (DTS) measurements. An obvious use of temperature measurements is to prevent overheating of the cable. Recently there has also been interest in using DTS measurements to detect deburial events by identifying cold spots on the cable route [9]. Deburial of the cable is undesirable as it can lead to additional mechanical strain on the cable and increase risk of damage by anchor drop. This places the cable at increased risk of failure. It would therefore be useful to develop thermal

This work was supported by AP Sensing GmbH, Böblingen, Germany. G. Callender, K.F. Goddard and J. A. Pilgrim are with the Tony Davies High Voltage Laboratory, University of Southampton, Southampton, SO17 1BJ, U.K. (e-mail: gmc1f17@ soton.ac.uk).

D.J. Ellis and J. K. Dix are with Ocean and Earth Science, University of Southampton, Southampton, SO14 3ZH, U.K.

M. Erdmann is with AP Sensing GmbH, Böblingen, Germany. models that can be used to provide an estimate of burial depth given the following inputs:

- Ambient temperature

- Sediment thermal properties

- Load data, in order to calculate cable losses

- DTS data

To ascertain the ambient temperature at cable depth the ambient ocean bottom temperature. These vary both spatially and temporally and can be ascertained from publically accessible: in situ wave buoys or tide gauge records; and/or sea surface temperature from satellite derived imagery. These approaches work well for well mixed ocean regimes where water temperature at the seabed is the same as the sea surface. Where seasonal thermal stratification occurs, or there is no in situ data a series of publically available ocean models are available (e.g. the MetOffice AMM15 model of the NW European Shelf [10]).

The sediment thermal properties (thermal conductivity and volumetric heat capacity) are all standardly measured, or derived, from pre-installation cable surveys [7]. These need to be captured for the top 1-3 m of the seabed from the site investigation data and utilized by the model. It is worth noting that calculating the burial depth from DTS data is more involved than the calculation of conductor temperature from DTS data. The reason for this is that the offset between the DTS fiber and the conductor is largely determined by heat transfer within the cable itself. In order to estimate burial depth it is also necessary to consider heat transfer within the marine environment which is more complex.

For a scenario with static load and without convective heat transfer, simple inversion of the formulae in IEC 60287-1, [11], would provide an estimate of burial depth. In real-world scenarios, load and temperature are never constant; so the influence of time varying load profiles were used to estimate burial depth using a predominantly analytical approach in [9]. However, this approach does not consider convective heat transfer through the sediment, i.e. heat being transferred away from the cable by the movement of water within the sediment. Previous work in the literature using both simulation and experimental measurements has confirmed that convection can take place in submarine environments and that for coarse sands (mean grain size $>500$ microns) it can even be the dominant form of heat transfer $[1,2,8]$. The simulations have solved both Darcy's law and the heat transfer equation in a two dimensional 'slice' geometry. This is a rigorous approach to consider heat transfer in a submarine environment and there can be confidence in the findings. However, a shortcoming of this approach is that bespoke finite element simulations typically require commercial software and an expert user. A further point is that the runtime, often in the order of minutes for a single scenario, could be prohibitive for a commercial application where large DTS datasets along an entire cable route must be analysed to determine conductor temperature and burial depth. 
This work introduces a one dimensional model to calculate transient temperature distributions for cables buried in marine sediments where heat transfer is taking place through both conduction and convection. The results obtained using this one dimensional thermal model are in close agreement with the more rigorous two dimensional model, and computation times are much smaller. Anyway, errors due to uncertainties in the sediment properties are likely to exceed the discrepancies between the thermal models; the sediment properties are subject to inhomogeneities, and are frequently insufficiently measured.

\section{Heat Transfer From Buried Submarine CABles}

In this section the mechanisms of heat transfer from buried submarine cables are introduced. The approach follows the work conducted in [1].

Within the cable the primary mechanism of heat transfer is by conduction. Within each domain of the cable the temperature, $\theta$, obeys

$$
C \frac{\partial \theta}{\partial t}-k \nabla^{2} \theta=Q
$$

where $C$ is the volumetric heat capacity of the material, $k$ is the thermal conductivity of the material and $Q$ is the heat sources within the material. At all boundaries between materials the temperature and the heat flux, $-k \vec{\nabla} \theta$, are continuous. These same conditions are also true at the cable surface at the boundary between the cable covering and the sediment. For HV AC submarine cables currents, and as a result joule heating, are present within the conductor, sheath and armor. Due to the alternating electric field dielectric losses are also present in the main insulation.

The sediment can typically be treated as a mixture of a solid phase and a liquid phase (pore water). In the absence of mass sources the conservation of pore water mass within the sediment requires that

$$
\vec{\nabla} \cdot\left(\rho_{1} \vec{u}\right)=0
$$

where $\rho_{\mathrm{l}}$ is the density of the pore water and $\vec{u}$ is the pore water velocity. The velocity of the pore water can be determined through Darcy's law

$$
\vec{u}=\frac{\kappa}{\mu}\left(\vec{g} \rho_{\mathrm{l}} \beta[\theta-\tilde{\theta}]-\vec{\nabla} p\right)
$$

where $\kappa$ is the permeability of the sediment, $\mu$ is the dynamic viscosity of the sediment, $p$ is the pressure, $\vec{g}$ is the gravitational acceleration vector, $\beta$ is the volume expansion coefficient of water, $\theta$ is the temperature and $\tilde{\theta}$ is a reference temperature. The choice of reference temperature will not impact the convective velocity, and consequently convective heat transfer, as the pressure gradient will adjust to balance any constant terms. Equation (3) makes a Boussinesq approximation that density variations are only considered when calculating buoyancy forces [1]. It is worth realizing that when temperature varies only with depth the pressure gradient will adjust to prevent any flow for a level seabed. The reason convection is observed in the simulations considered in this work is that the cable will create temperature gradients that are not normal to the level seabed. In reality additional hydrodynamic processes may create local pore water convection along a cable route. A seabed that is not level could allow convective processes to take place under ambient conditions.
The temperature distribution in the sediment can be determined by solving the heat transfer equation

$$
C_{\mathrm{v}_{\mathrm{eff}}} \frac{\partial \theta}{\partial t}+C_{\mathrm{vl}} \vec{u} \cdot \vec{\nabla} \theta-k_{\mathrm{eff}} \nabla^{2} \theta=0
$$

where $C_{\mathrm{v}_{\text {eff }}}$ is the effective volumetric heat capacity of the sediment, $C_{\mathrm{v} l}$ is the volumetric heat capacity of the pore water and $k_{\text {eff }}$ is the effective thermal conductivity of the sediment.

It is quite reasonable to treat the seabed surface as an isotherm, unaffected by the cable. The reason for this is the very high rate of heat transfer due to seawater convection combined with the high volumetric heat capacity of water. It should also be noted that ambient seabed temperatures can have a strong dynamic component and in certain regions may vary by as much as $10^{\circ} \mathrm{C}$ over a year [12].

\section{THERMAL MODELS}

Two different thermal models are used in this work. The first is a two dimensional simulation which is treated as the baseline to which the fast one dimensional model can be compared. The cable considered in this work is a HV threecore AC windfarm export cable, identical to a model previously introduced in the literature [8]. The dimensions of the cable model geometry are provided in Table 1, the cable material thermal properties are taken from [1], they are provided in Table 2

TABLE I

WIND FARM EXPORT CABLE GEOMETRY

\begin{tabular}{|c|c|c|}
\hline Component & Material & $\begin{array}{c}\text { Outer Diameter } \\
{[\mathbf{m m}]}\end{array}$ \\
\hline Conductor & Copper & 34.3 \\
\hline $\begin{array}{c}\text { Conductor } \\
\text { Screen }\end{array}$ & XLPE & 37.3 \\
\hline Insulation & XLPE & 71.3 \\
\hline $\begin{array}{c}\text { Insulation } \\
\text { Screen }\end{array}$ & XLPE & 74.3 \\
\hline Swelling Tape & Polymeric & 77.3 \\
\hline Sheath & Lead & 81.9 \\
\hline Oversheath & PE & 86.3 \\
\hline Filler & PP & 186.0 \\
\hline Binder tape & Polymeric & 189.8 \\
\hline Armour & Steel & 201.0 \\
\hline Serving & PP & 210.0 \\
\hline
\end{tabular}

TABLE II

MATERIAL THERMAL PROPERTIES [1]

\begin{tabular}{|c|c|c|}
\hline Material & $\begin{array}{c}\text { Thermal } \\
\text { Conductivity } \\
{[\mathbf{W} / \mathbf{m K} \text { ] }}\end{array}$ & $\begin{array}{c}\text { Volumetric } \\
\text { Heat Capacity } \\
{\left[\mathbf{M J} / \mathbf{m}^{\mathbf{3}} \mathbf{K}\right]}\end{array}$ \\
\hline Copper & 400 & 3.49 \\
\hline XLPE & $1 / 3.5$ & 1.98 \\
\hline Polymeric & 0.2 & 1.26 \\
\hline Lead & 35.3 & 1.47 \\
\hline PE & $1 / 3.5$ & 1.26 \\
\hline PP & 0.2 & 1.26 \\
\hline Steel & 18 & 3.82 \\
\hline
\end{tabular}

Heat sources used in the thermal models are taken from the IEC standard [13]. It is acknowledged that present research is providing recommendations for altering these losses $[14,15]$. 
However, this work is fundamentally concerned with the thermal modelling of the burial environment. Adjustments to cable losses are unlikely to impact the findings of this work as we are concerned with heat transfer outside of the cable. The dynamic viscosity of the pore water $\mu$ is set to $0.001382 \mathrm{~Pa} \mathrm{~s}$, the density of the pore water $p_{\mathrm{l}}$ is set to $1023 \mathrm{~kg} / \mathrm{m}^{3}$ and the thermal expansion coefficient $\beta$ is set to $0.00021 \mathrm{~K}^{-1}[16,17]$. The following features of the burial environment are varied in the different scenarios considered in this paper:

- $\quad$ Sediment properties $\left(k_{\text {eff }}\right.$ and $\left.\kappa\right)$

- Burial depth, taken as distance between the center of the cable and the seabed

The values used in each simulation will be explicitly stated. The effective volumetric heat capacity of the sediment, $C_{\mathrm{v} \text { eff }}$, is set to $3 \mathrm{MJ} / \mathrm{m}^{3} \mathrm{~K}$, which is a typical value for quartz sand sediments [18].

\section{A. Two Dimensional Thermal Model}

A two dimensional finite element simulation is constructed using the commercial software Comsol [19]. Cable components are explicitly represented as a series of circles within the geometry. The full domain is a rectangular box which extends $25 \mathrm{~m}$ below the cable and $25 \mathrm{~m}$ to the right of the cable. Due to the symmetry of the model a zero heat flux boundary is employed on the $y$ axis. The model geometry is provided in Figure 1.

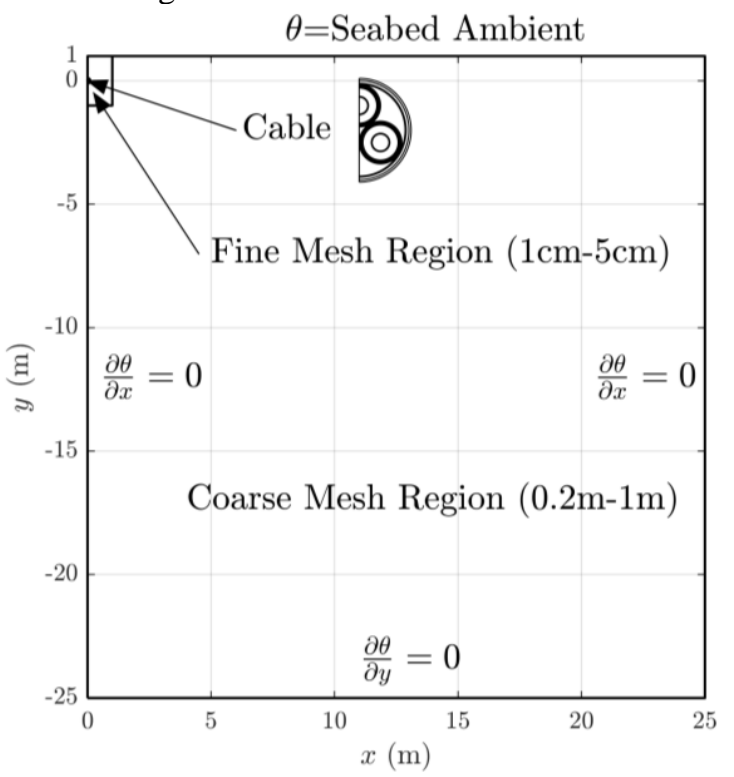

Fig. 1. Schematic of the two dimensional finite element simulation model.

The governing equations for heat transfer, (1) and (4), are solved in the cable and sediment domains respectively to calculate the temperature. The pressure, from which the convective velocity is determined, is only calculated in the sediment. Within components containing heat sources, such as the conductors, the losses are treated as uniform.

The temperature at the seabed boundary is set to the ambient seabed temperature, and the pressure is fixed. At the three other external boundaries zero normal convective velocity, $\vec{u} \cdot \vec{n}=0$, and zero normal heat flux, $\vec{n} \cdot k \vec{\nabla} \theta=0$, boundary conditions are enforced. The normal convective velocity is also set to zero at the surface of the cable.

The simulation was performed on an unstructured triangular mesh with quadratic order shape elements. Mesh refinement was performed by restricting the maximum element size, which is defined as the largest distance between two vertices of each triangular element. For all meshes used within this work the temperature was found to vary by less than $0.1^{\circ} \mathrm{C}$ when the number of mesh elements was increased by a factor of two. The model dependency on far-field boundaries is negligible and has been investigated previously [1].

\section{B. One Dimensional Thermal Model}

A simple thermal network model of nodes with a thermal capacitance connected by thermal resistances is used to capture heat transfer within the cable. This is an established approach in the literature, e.g. $[6,20]$, and as such it is not discussed in detail here, a schematic is provided in Figure 2. Fundamentally the contribution of this work is to consider heat transfer within the burial environment and the methodology for this is independent of the cable thermal model. The number of nodes in the thermal network was set to be 1 for each cable component for static calculations and 5 for each cable component for dynamic calculations.

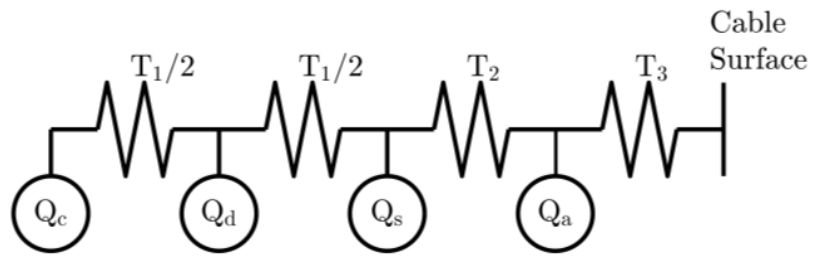

Fig. 2. Schematic of the cable thermal network model. Heat sources from the conductor $Q_{\mathrm{c}}$, dielectric $Q_{\mathrm{d}}$, sheath $Q_{\mathrm{s}}$ and armour $Q_{\mathrm{a}}$ are taken from the IEC standards [11]. Thermal resistances of the dielectric $T_{1}$, bedding $T_{2}$ and outer covering $T_{3}$ are taken from the IEC standards [13]. A novel approach is derived in this paper for calculating convective heat transfer in marine sediments between the cable surface and the seabed.

The IEC standards, [13], provide thermal resistance formulae for bedding region of three-phase export cable designs. However, existing work in the literature, [21], has demonstrated that they may introduce errors of approximately $5{ }^{\circ} \mathrm{C}$ when compared to finite element simulations. As such the thermal resistance of the bedding, $T_{2}$, for the export cable thermal network was determined from the two dimensional simulation by calculating the average temperature difference between the sheath covering the inner diameter of the armour.

When heat transfer takes place by conduction only it is reasonable to represent the burial environment as a one dimensional axisymmetric model which can be used with an inner boundary at the radius of the cable and an outer boundary at ambient conditions located at twice the burial depth, $B$, of the cable. This is essentially assuming that the approximation to the thermal resistance of the soil, $T_{4}$, provided in the IEC standards is an exact representation of the burial environment. It is desirable to construct the model from this one dimensional axisymmetric foundation as it will provide accurate calculations of temperature for a conduction only scenario. However, it is necessary to use a two dimensional model if the conservation of volume, (2), is to still be valid. To this end it is assumed that the temperature and pressure can be described by the following functions within the sediment

$$
\begin{gathered}
p(r, \phi, t)=p_{1}(r, t) \cos \phi \\
\theta(r, \phi, t)=\theta_{0}(r, t)+\theta_{1}(r, t) \cos \phi
\end{gathered}
$$

where $\phi$ is the polar angle. The coordinate system is orientated such that $r=0$ corresponds to the centre of the 
cable and $\phi=0$ points in the $y$ direction. The assumption in (5) is that the pressure gradient, and consequently the convective velocity, has a radial component which is dependent on the cosine of the polar angle and an angular component which is dependent on the sine of the polar angle. This will match the physical behaviour of pore water moving upwards driven by buoyancy. The temperature, $\theta$, is set equal to a first order expansion in $\cos n \phi ; \sin n \phi$ terms can be neglected due to symmetry. The model can be regarded as a fully two dimensional model in a polar coordinate system that only considers the leading order slowly varying angular component.

It is also assumed that the $r$ component of the convective velocity, $u_{r}$, is dependent on the cosine of the polar angle and has the following form

$$
u_{r}=v \cos \phi
$$

where the variable $v(r, t)$ has been introduced as follows

$$
v=\frac{\kappa}{\mu}\left(g \rho_{\mathrm{l}} \beta\left[\theta_{0}-\theta_{B \mathrm{amb}}\right]-\frac{\partial p_{1}}{\partial r}\right) .
$$

The temperature rise term has been modified by setting it to the temperature rise above the ambient temperature at burial depth of the cable, $\theta_{B \text { amb }}$. This ensures that the convective velocity is negligible when there are no additional heat sources and the temperature gradient is due to ambient temperature variations in the downwards direction through the sediment. In a fully two dimensional model this result is ensured. To find $\theta_{\mathrm{B} \text { amb }}$ a simple one dimensional conduction only heat transfer problem is solved

$$
C_{\mathrm{v}_{\mathrm{eff}}} \frac{\partial \theta_{\mathrm{amb}}}{\partial t}-k_{\mathrm{eff}} \frac{\partial^{2} \theta_{\mathrm{amb}}}{\partial y^{2}}=0 .
$$

It is necessary to solve for the diffusion of ambient temperature downwards through the sediment due to the variation in temperature throughout the year. It should be noted that in some regions annual variations may be as high as $10^{\circ} \mathrm{C}$ [12]. For a coordinate system with origin at the centre of the cable $\theta_{B \mathrm{amb}}(\mathrm{t})=\theta_{\mathrm{amb}}(\mathrm{y}=0, \mathrm{t}) . \theta_{\mathrm{amb}}$ is set equal to the ambient seabed temperature profile at $y=B$.

The conservation of volume, (2), means that the angular component of the convective velocity $u_{\phi}$ is

$$
u_{\phi}=-\frac{\partial(r v)}{\partial r} \sin \phi
$$

Equation (2) can also be used to write the governing equation for pressure as

$$
r \frac{\partial^{2} p_{1}}{\partial r^{2}}+\frac{\partial p_{1}}{\partial r}-\frac{p_{1}}{r}-r \rho_{l} g \beta \frac{\partial \theta_{0}}{\partial r}=0 .
$$

The boundary conditions for pressure are $u_{r}=0$ at $r=\frac{D}{2}$ and $p=0$ at $r=2 B$. The boundary condition at $r=D / 2$ means that pore water will flow around the cable at the cable surface. The seabed boundary condition constrains the pressure field. Changing the constant value of the pressure at the seabed will not impact the convective velocity.

Substituting (7) and (8) into the heat transfer equation for the sediment (4) yields

$$
\begin{array}{cc}
C_{\mathrm{v}_{\text {eff }}} \frac{\partial \theta_{0}}{\partial t}+C_{\mathrm{v}_{\mathrm{eff}}} \cos \phi \frac{\partial \theta_{1}}{\partial t} & + \\
C_{\mathrm{v}_{\mathrm{l}}} v \cos \phi\left[\frac{\partial \theta_{0}}{\partial r}+\frac{\partial \theta_{1}}{\partial r} \cos \phi\right] & + \\
\frac{k_{\mathrm{eff}}}{r}\left[\frac{\partial(r v)}{\partial r} \frac{\theta_{1}}{r} \sin ^{2} \phi\right. & +
\end{array}
$$

Isolating terms by powers of $\cos \phi$, and setting the quadratic powers of sin and cos to their average value of 0.5 results in two equations

$$
\begin{gathered}
C_{\mathrm{v}_{\mathrm{eff}}} \frac{\partial \theta_{0}}{\partial t}+\frac{1}{2 r} C_{\mathrm{v}_{\mathrm{l}}} \frac{\partial}{\partial r}\left(r v \theta_{1}\right)=\frac{k_{\mathrm{eff}}}{r}\left[\frac{\partial}{\partial r}\left(r \frac{\partial}{\partial r} \theta_{0}\right)\right] \\
C_{\mathrm{v}_{\text {eff }}} \frac{\partial \theta_{1}}{\partial t}+C_{\mathrm{v}_{\mathrm{l}}} v \frac{\partial \theta_{0}}{\partial r}=\frac{k_{\mathrm{eff}}}{r}\left[\frac{\partial}{\partial r}\left(r \frac{\partial}{\partial r} \theta_{1}\right)-\frac{\theta_{1}}{r}\right] .
\end{gathered}
$$

Choosing boundary conditions for $\theta_{0}$ and $\theta_{1}$, which are required to solve (13) and (14), is not trivial. At the cable surface it is assumed that the temperature is uniform around the entire cable. This means $\theta_{1}=0$ at $r=D / 2 ; \theta_{0}$ at $r=$ $D / 2$ is set to ensure continuity of heat flux and temperature. This has the added benefit of allowing the use of a purely radial thermal model to capture heat transfer the cable surface. An example of a heat transfer model is a simple thermal network model, [20], which as previously mentioned is the approach taken in this work. At $r=2 B$ it is decided to set $\theta_{0}$ to the ambient seabed temperature and $\theta_{1}=0$. Fundamentally these choices are justified due to the close agreement between this model and a fully two dimensional simulation.

The resulting model is quasi one dimensional, in that there are two governing equations to solve for the temperature, (13) and (14) which depend only on the $r$ coordinate. There are in total four governing equations, (9), (11), (13) and (14), which are derived in this paper and represent the novel contribution of this work. These equations are all solved using second order central finite difference schemes in space with backward Euler time stepping. The grid size and time step size were reduced until simulation results were found to change by less than $0.1{ }^{\circ} \mathrm{C}$ when the grid size and time step size were both reduced by a factor of 2 .

\section{MODEL COMPARISON}

In this section a comparison of the two dimensional model against the low cost one dimensional model is undertaken. Both static and dynamic scenarios are considered; the level of agreement between the models is consistently within $1.5^{\circ} \mathrm{C}$. Throughout this section the conductor temperature of the two dimensional model is calculated as the average temperature across all conductors.

\section{A. Continuous Loads}

A comparison of static continuous load scenarios are considered first. The seabed ambient temperature is taken as a constant value of $12^{\circ} \mathrm{C}$ for all cases considered in this section.

In Figure 3 the conductor temperature calculated using both models, and the corresponding error, is compared for different sediment thermal conductivities over a range of permeabilities with the cable at a burial depth of $1 \mathrm{~m}$. The input load has been set such that the conductor temperature is $90^{\circ} \mathrm{C}$ in a low permeability sediment where heat transfer is 
dominated by conduction. The permeability range considered is $10^{-13} \mathrm{~m}^{2}$ to $10^{-9} \mathrm{~m}^{2}$. Permeabilities below $10^{-13} \mathrm{~m}^{2}$ may occur regularly in nature [7], however, it was found that convective heat transfer is negligible even at $10^{-13} \mathrm{~m}^{2}$, so there is no purpose in including lower values in this analysis. Sediment thermal conductivities are varied between $1 \mathrm{~W} / \mathrm{mK}$ and $3 \mathrm{~W} / \mathrm{mK}$ which may be regarded as typical values for clays, peats and carbonate sands $(1 \mathrm{~W} / \mathrm{mK})$ and quartz sands (2-3 W/mK) respectively [7]. It should be realised that in practice a range of thermal conductivities may be expected for all sediment types.

Close agreement was observed between the one and two dimensional models, with a maximum error below $1.5^{\circ} \mathrm{C}$ over the parameter sweep considered here. The absolute difference is displayed in Figure 3c. The largest errors occurred in the transition from the thermal conduction dominated regime to the convection dominated regime and in particular where thermal conductivities are particularly low (a scenario restricted to coarse carbonate sands. In this region the one dimensional model typically calculated slightly lower conductor temperatures than the two dimensional model. Smaller rises above ambient conditions will lead to lower model errors simply due to the smaller temperature difference. However, cables are typically rated to reach conductor temperatures close to $90^{\circ} \mathrm{C}$ using worst case sediment thermal conductivities and IEC 60287 which neglects convective heat transfer $[11,13]$. Therefore such errors are more likely to be indicative of real world operation. It is worth noting that if convective heat transfer is neglected, significant over predictions of conductor temperature could be expected.

It has been demonstrated previously that convective heat transfer will lead to a plume rising above the cable to the seabed [1,8]. In Figure 4 a comparison is made of the temperature profiles for both models. It can be seen that there is reasonable agreement in terms of the broad temperature profiles with a clear convective plume in both scenarios. In order to quantitatively compare temperature distributions between the one dimensional and two dimensional models within the sediment it is necessary to construct a conformal mapping between the two domains. This is beyond the scope of this paper and is an area of future research.

In terms of ampacity, the presence of convective heat transfer allows for significant increases. Table 3 shows the results of ampacity calculations for three generic marine sediments [7]: high permeability, very coarse, quartz sands; low permeability marine clay; and high permeability, coarse, carbonate sands. The first two are very typical of mid- high latitude continental shelves where much of the current windfarm expansion is occurring. The latter is more common on lower latitude shelves (such as Australia) and represents a material that can have high permeability but low thermal conductivity. The load required for the conductor temperature of the submarine cable considered in this work to reach $90^{\circ} \mathrm{C}$ was calculated using: the two dimensional model, the one dimensional model and an IEC 60287 based approach, $[11,13]$, which neglects convective heat transfer. The burial depth of the cable was fixed at $1 \mathrm{~m}$, the ambient temperature was set to $12^{\circ} \mathrm{C}$. In the low permeability marine clays conduction is the sole mode of heat transfer and so the Ampacity for both 2D, 1D and IEC models are the same. However, the high permeability quartz sediments can support over c. 350A more than the standard IEC case. The discrepancy is even greater in the carbonate sediments (c. 500A) as the low natural thermal conductivity of the carbonate grains gives a very low ampacity for the conventional IEC calculation.
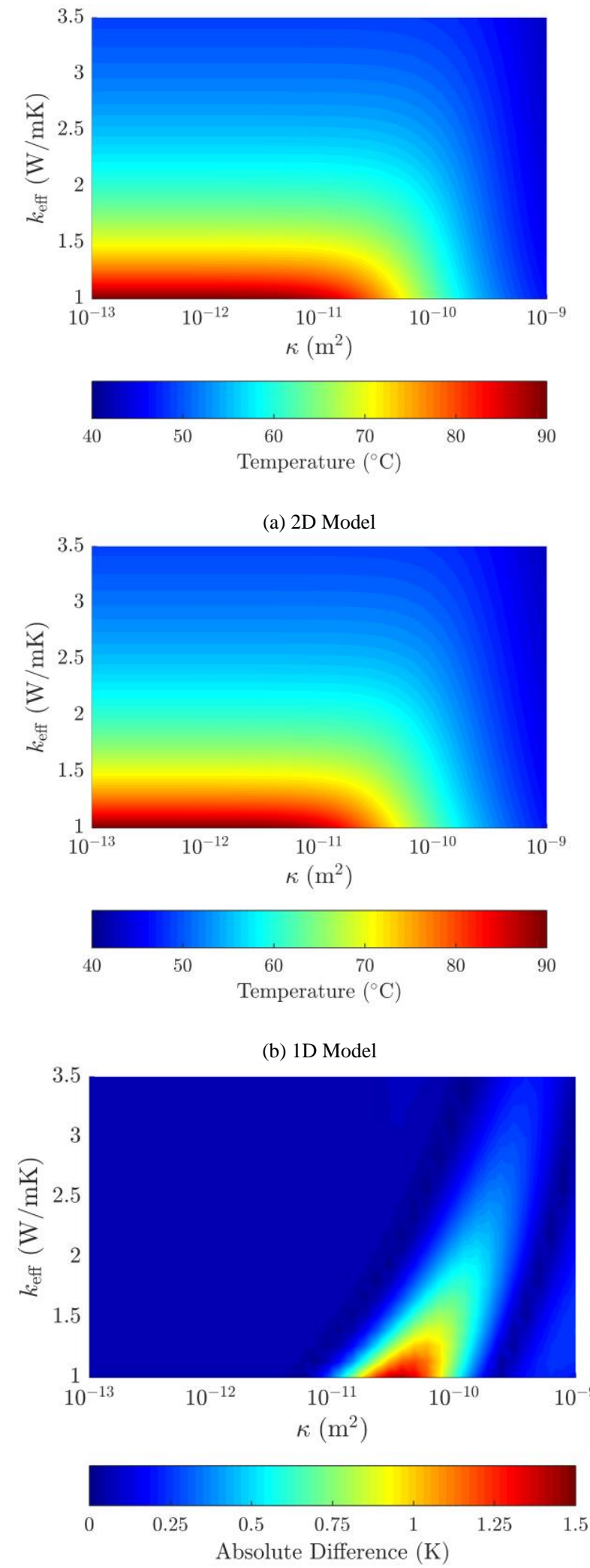

(c) Model Difference

Fig. 3. Comparison of conductor temperature profiles for the: (a) two dimensional model, (b) one dimensional model and (c) model difference. The cable is buried at $1 \mathrm{~m}$, the ambient temperature is $12^{\circ} \mathrm{C}$. The load is set such that the conductor temperature is $90^{\circ} \mathrm{C}$ in sediments with low thermal conductivity and permeability. 

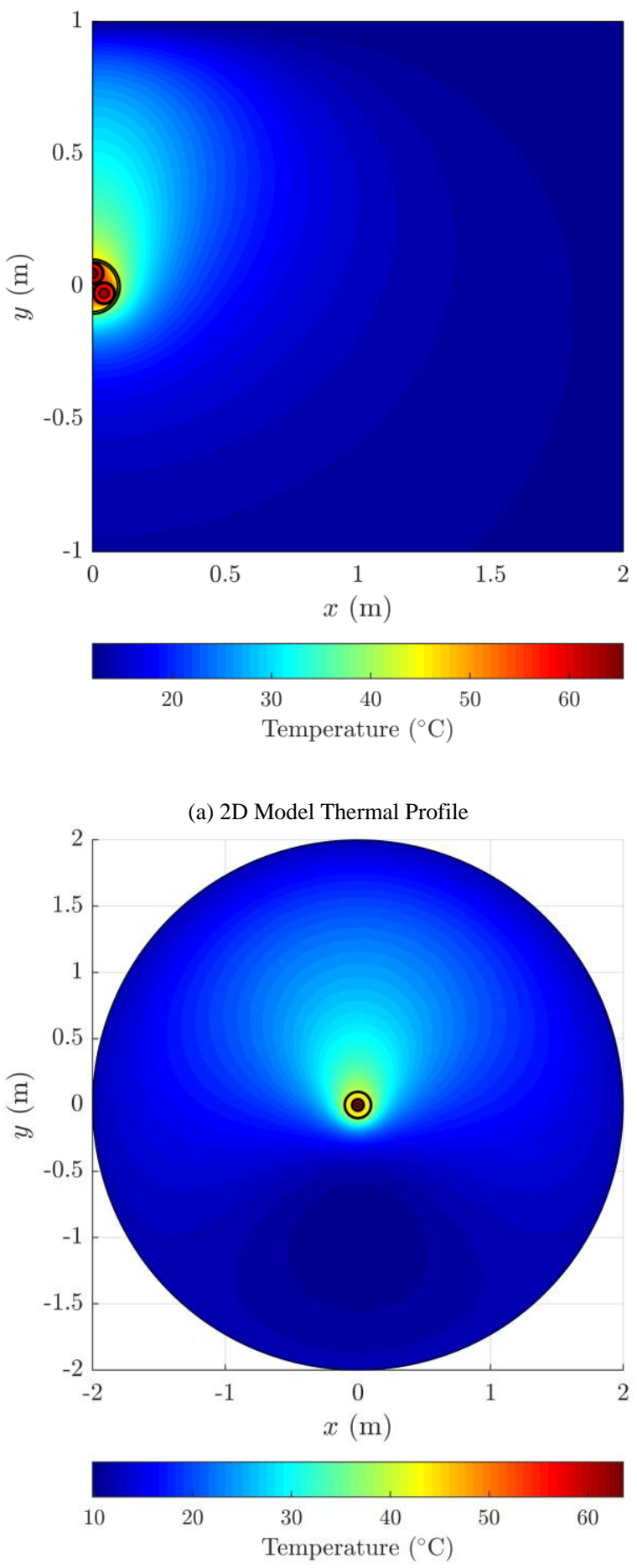

(b) 1D Model Thermal Profile

Fig. 4. Temperature profiles for the: (a) two dimensional model and (b) one dimensional model. The cable is buried at $1 \mathrm{~m}$, the ambient temperature is set to $12^{\circ} \mathrm{C}$ and the sediment thermal conductivity is $2 \mathrm{~W} / \mathrm{mK}$. The permeability is $10^{-10} \mathrm{~m}^{2}$, the load is set such that in low permeability sediments the conductor temperature is $90^{\circ} \mathrm{C}$.

The agreement between the one dimensional model and two dimensional model could potentially be improved by introducing a cosine dependent term into the temperature field inside the cable itself. This extension was not considered in this work because the agreement between the one dimensional and two dimensional models is still reasonable and it would exclude the use of a single thermal network model of the cable which is an established technique in the literature.
TABLE III

CABLE AmpaCities In A Range Of Sediments CalCUlated Using DIFFERENT THERMAL MODELS

\begin{tabular}{|c|c|c|c|}
\hline \multirow{2}{*}{ Sediment } & \multicolumn{3}{|c|}{ Ampacity [A] } \\
\cline { 2 - 4 } & 2D & 1D & IEC \\
\hline $\begin{array}{c}\text { Very Coarse Quartz Sands } \\
k_{\text {eff }}=2.2 \mathrm{~W} / \mathrm{mK} \\
\kappa=4.7 \times 10^{-9} \mathrm{~m}^{2}\end{array}$ & 1453 & 1450 & 1104 \\
\hline $\begin{array}{c}\text { Marine Clay } \\
k_{\text {eff }}=1.2 \mathrm{~W} / \mathrm{mK}\end{array}$ & 923 & 923 & 923 \\
$\kappa=2.75 \times 10^{-13} \mathrm{~m}^{2}$ & & & \\
\hline $\begin{array}{c}\text { Carbonate Sand } \\
k_{\text {eff }}=1.1 \mathrm{~W}^{-} \mathrm{mK} \\
\kappa=2.5 \times 10^{-9} \mathrm{~m}^{2}\end{array}$ & 1394 & 1395 & 896 \\
\hline
\end{tabular}

Another observation from the two dimensional models is that for sediments where conductive heat transfer dominates the conductor temperature of the power cores furthest from the seabed is higher than the conductor temperature of the power core nearest the seabed. This is as expected due to the relative proximity to the ambient seabed aiding conductive heat transfer. However, when convective heat transfer becomes significant this situation reverses, with the power cores furthest from the seabed becoming colder than the power core nearest the seabed due to the warming water flowing circumferentially around the cable. In reality the helical twist of the power cores along the cable route is likely to 'average out' this effect. A further point is that the formation of a convective plume will lead to higher temperatures in the vicinity of the seabed, which may have implications for environmental controls and the " $2 \mathrm{~K}$ " rule [12]. The mapping of temperatures in the sediment from the one dimensional model domain to the two dimensional domain is an area of current research.

For high permeability sediments it has been demonstrated that cable temperatures are insensitive to burial depth [8]. In fact, cable temperatures are actually slightly higher at lower burial depths for sediments with a high level of convective transfer, as shown in Figure 5. The reason for this is that at deeper burial depths the convective plume is larger, due to greater distance between the cable at the seabed. The presence of a larger plume promotes greater circumferential flow and results in a greater degree of cooling. The one dimensional model which only considers first order cosine terms, and uses a different description of the burial environment, was not able to capture this high order effect.

A final point to make regarding the static scenario calculations is on the relative computational speed of the one dimensional and two dimensional models. In order to solve for a highly permeable sediment the solution time of the one dimensional model was approximately 0.6 seconds, whereas the two dimensional model was approximately 21 seconds. Both models were run on a desktop with $32 \mathrm{~GB}$ of RAM with Intel $17-47903.6 \mathrm{GHz}$ processors, and the stated solution times are the average of 20 runs. It is acknowledged that the implementation of the one dimensional model was not highly optimized and the two dimensional model used an unstructured triangular mesh, so it is difficult to systematically perform mesh coarsening to improve computational speed. However, it may still be robustly concluded that the one dimensional model offers significant savings in computational time. 


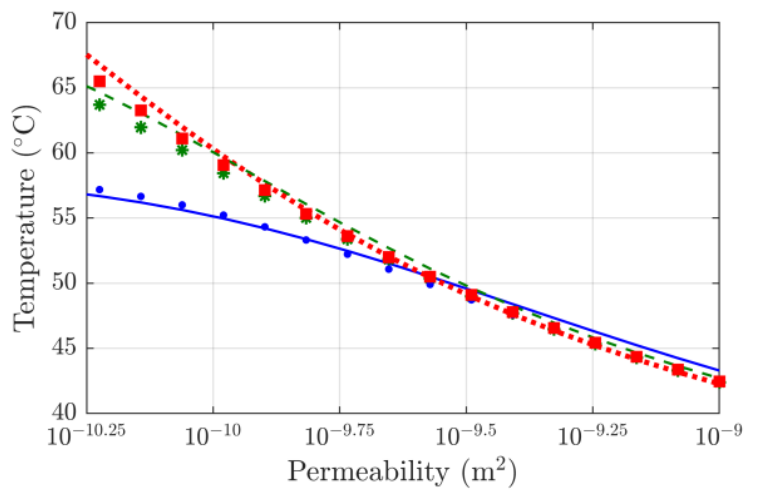

$2 \mathrm{D} B=0.25 \mathrm{~m} \cdots--2 \mathrm{D} B=0.5 \mathrm{~m} \quad \ldots \ldots . .2 \mathrm{D} B=1 \mathrm{~m}$ - $1 \mathrm{D} B=0.25 \mathrm{~m} \quad * \quad 1 \mathrm{D} B=0.5 \mathrm{~m} \quad$ - $\quad 1 \mathrm{D} B=1 \mathrm{~m}$

Fig. 5. Conductor temperatures for different burial depths using both the one dimensional and two dimensional model. The ambient temperature is set to $12^{\circ} \mathrm{C}$ and the sediment thermal conductivity is $1 \mathrm{~W} / \mathrm{mK}$. The load is set such that the conductor temperature at low permeabilities (not included in the figure) at $1 \mathrm{~m}$ burial depth is $90^{\circ} \mathrm{C}$. For highly permeable sediments the conductor temperature was found to decrease at higher burial depths in the two dimensional model.

\section{B. Dynamic Loads}

Wind farm operators may choose to utilize dynamic ratings to enhance circuit capacity [22]. Furthermore, the analysis of operational data, for example to determine burial depth changes, requires transient temperature simulations. It is therefore necessary to test whether the one dimensional model is capable of capturing these dynamics.

A comparison of conductor temperatures for different burial depths during a 6 hour dynamic rating test, is provided in Figure 6. The initial condition is a static solution at $50 \%$ of the continuous load. The rating current was calculated assuming no convective heat transfer, in accordance with the IEC standards [13], such that the conductor temperature reached $90^{\circ} \mathrm{C}$ after 6 hours at the rating current. The one dimensional model was within $1{ }^{\circ} \mathrm{C}$ of the two dimensional model in all of the scenarios considered. For the sake of brevity only the results for a convective sediment, with a permeability of $10^{-10} \mathrm{~m}^{2}$ are provided.

Due to the relatively short 6 hour period of the rating load the convective plume does not have time to become fully established and as such the influence of convective cooling is relatively minimal. It is important to see that similar dynamics are observed for both the one dimensional and two dimensional models. The one dimensional model was found to calculate temperatures which were slightly lower than the two dimensional model except for the times near the end of the rating current for shallow burial depths.

It is also of interest to consider the capability of the model to capture transient temperature profiles that may be experienced during operation where the load will vary rapidly. A 16 year-long synthetic wind time series was used to produce a load time series with dynamics indicative of wind farm operation and thus representative of operational loads the export cable may experience. A period of 100 days where the load data was relatively high, with an $80 \%$ load factor, was selected to test the thermal models. The conductor temperature time series from two sediments with the same thermal conductivity, but different permeabilities, are provided in Figure 7. The initial condition in both models was the RMS load of the entire 16 year time series.

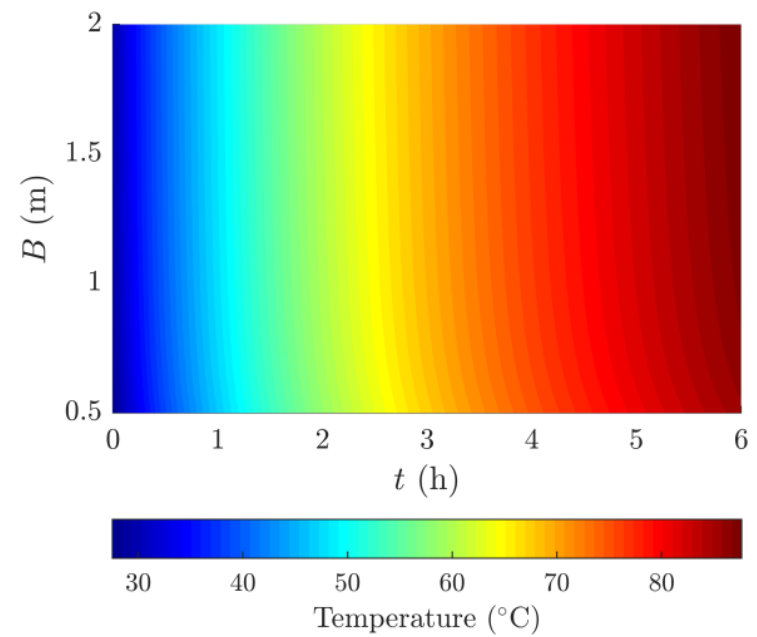

(a) 2D Model

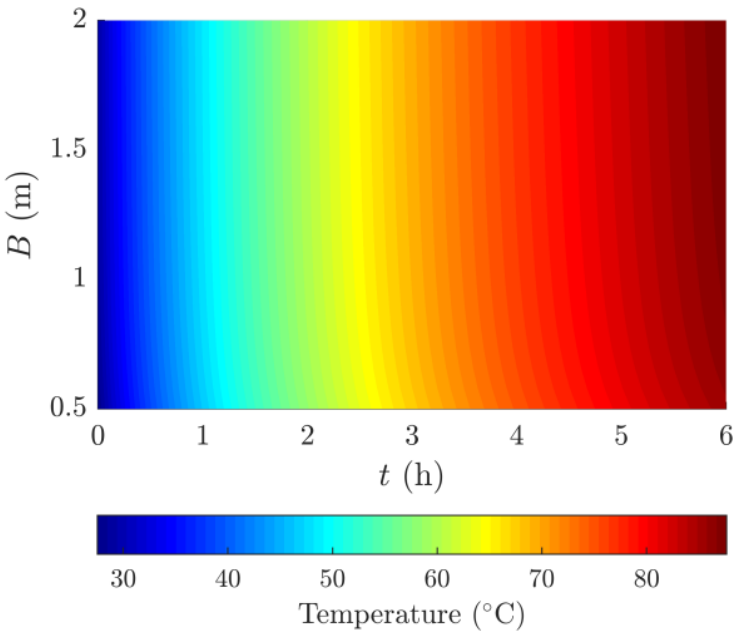

(b) $1 \mathrm{D}$ Model

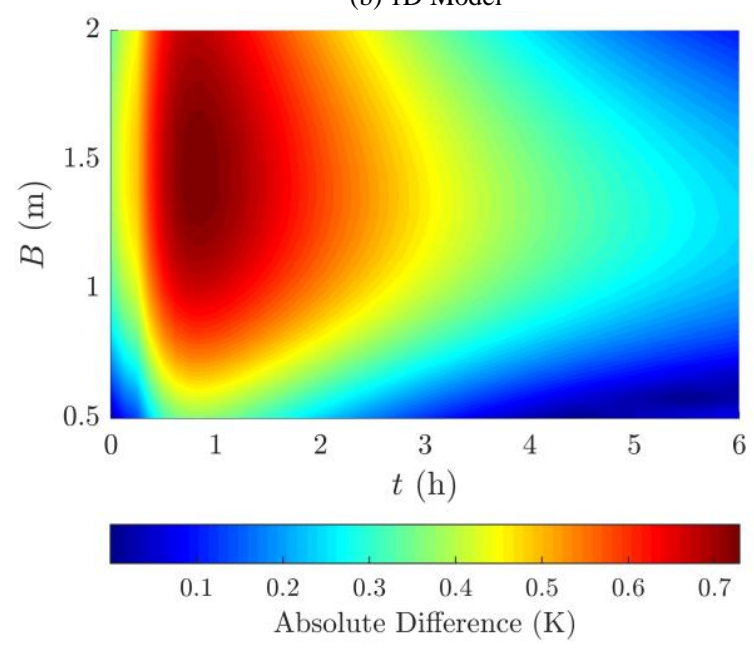

(c) Model Differences

Fig. 6. Comparison of conductor temperatures during dynamic ratings for burial depths during a 6 hour rating transient: (a) two dimensional model, (b) one dimensional model and (c) model difference. The ambient temperature is set to $12^{\circ} \mathrm{C}$, the sediment thermal conductivity is $1 \mathrm{~W} / \mathrm{mK}$ and the sediment permeability is set to $10^{-10} \mathrm{~m}^{2}$. The initial load is $50 \%$ of the continuous load which results in static conductor temperatures of $90^{\circ} \mathrm{C}$ in low permeability sediments. The same dynamic load is used in all cases considered. 


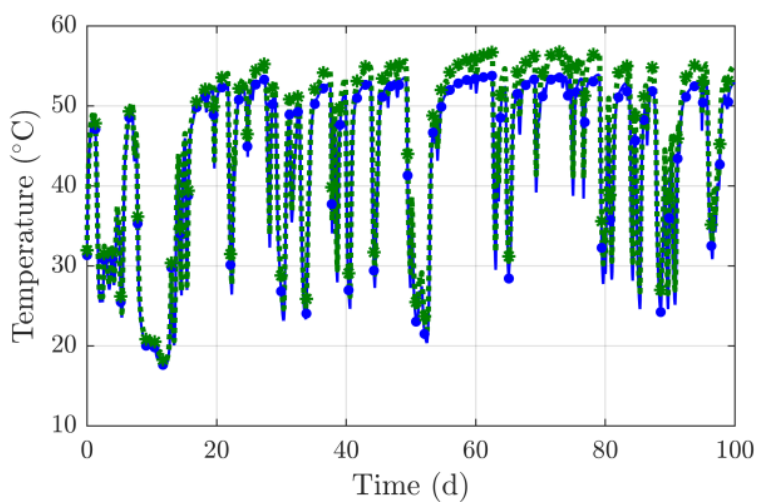

$2 \mathrm{D} \kappa=10^{-10} \mathrm{~m}$
1D $\kappa=10^{-10} \mathrm{~m}$
1D

(a) Conductor Temperature Profiles

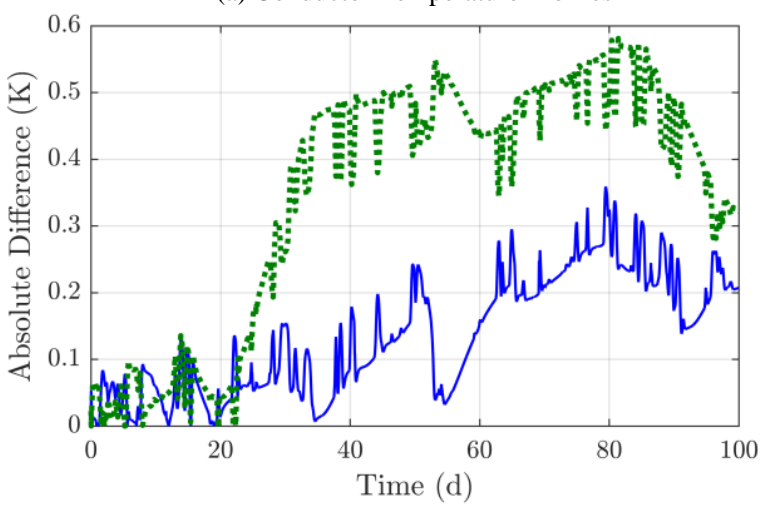

$\leftarrow=10^{-10} \mathrm{~m} \cdots \cdots \cdots \kappa=10^{-13} \mathrm{~m}$

(b) Model Differences

Fig. 7. Conductor temperature profiles calculated using a highly dynamic load over a 100 day period for different sediment permeabilities: (a) conductor temperature time series and (b) model difference. The ambient temperature is set to $12^{\circ} \mathrm{C}$, the sediment thermal conductivity is $2 \mathrm{~W} / \mathrm{mK}$.

The results show that the one dimensional model is able to successfully capture highly dynamic conductor temperature profiles for sediments with a high permeability. It can be seen that during the beginning of the simulation the conductor temperatures are fairly similar for both permeabilities due to the relatively low initial load. During the 100 day period the relatively high load leads to a divergence with the additional heat transfer due to convection at the high permeabilities leading to lower cable temperatures.

The runtime of the one dimensional model is significantly lower than the two dimensional model for dynamic scenarios. Each time step in the one dimensional model takes approximately $0.01 \mathrm{~s}$, whereas in the two dimensional model each time step takes approximately $10 \mathrm{~s}$. The desktop configuration has been discussed previously

\section{CONCLUSION}

The fundamental contribution of this work is the introduction of a relatively simple thermal model which is capable of capturing convective and conductive heat transport in submarine cables. For the scenarios tested the typical error was below $1.5^{\circ} \mathrm{C}$. For a static solution the runtime was typically $0.6 \mathrm{~s}$, which is a significant improvement over the $21 \mathrm{~s}$ required for the comparable two dimensional simulation. For a dynamic scenario the saving in computational time is approximately 3 orders of magnitude. It is likely that optimization of the one dimensional model implementation could further decrease its computational cost.

Convective heat transfer can have a significant impact on cable ratings. It has been demonstrated that errors in excess of $30^{\circ} \mathrm{C}$ could be expected if a thermal model which only considers conduction and neglects convection, for example IEC 60287 [11], is used. This results in cable ratings which could be $50 \%$ higher in highly permeable sediments compared to sediments with a similar thermal conductivity but a low permeability. Put differently, methods that do not include convective heat transfer may underestimate cable ampacity by $30-55 \%$, this is shown explicitly in Table 3. Furthermore, for convective scenarios the sensitivity of cable temperatures to burial depth is greatly reduced. Considering convective heat transport is therefore vitally important to properly interpret temperature data from submarine cables in highly permeable sediments.

The low computational cost model introduced in this work allows for large DTS datasets to be rapidly analysed for a number of applications including: dynamic rating; burial depth prediction; and long-term cable performance.

\section{REFERENCES}

[1] T. J. Hughes, "Environmental controls on the state of HV cables under the seafloor", Ph.D. dissertation, Dept. Ocean \& Earth Science, Southampton Univ., Southampton, UK, 2016.

[2] C. J. Emeana, T. J. Hughes, J. K. Dix, T. M. Gernon, T. J. Henstock, C. E. L. Thompson and J. A. Pilgrim, "The thermal regime around buried submarine high-voltage cables," Geophys. J. Int., vol. 206, no. 2, pp. 1051-1064, 2016.

[3] C. Macilwain, "Energy: Supergrid," Nature, vol. 468, no. 7324, pp 624-625, 2010

[4] J. de Decker and A. Woyte, "Review of the Various Proposals for the European Offshore Grid," Renew. Energy, vol. 49, pp. 58-62, 2013.

[5] F. de Leon and G. J. Anders, "Effects of Backfilling on Cable Ampacity Analyzed With the Finite Element Method," IEEE Trans. on Power Del., vol. 23, no. 2, pp. 537-543, 2008.

[6] G. J. Anders, Rating of Electric Power Cables in Unfavourable Thermal Environments, Hoboken, NJ: John Wiley \& Sons Inc, 2005.

[7] J. K. Dix, T. J. Hughes, C. J. Emeana, J. A. Pilgrim, T. M. Gernon, C. E. L. Thompson and M. E. Vardy, "Substrate controls on the lifetime performance of marine HV cables," in OSIG 2017, London, UK, 2017, pp. 88-107.

[8] T. J. Hughes, T. J. Henstock, J. A. Pilgrim, J. K. Dix, T. M. Gernon and C. E. L. Thompson, "Effect of Sediment Properties on the Thermal Performance of Submarine HV Cables," IEEE Trans. Power Del., vol. 30, no. 6, pp. 2443-2450, 2015.

[9] J. Lux, M. Olschewski, P. Schäfer and W. Hill, "Real-Time Determination of Depth of Burial Profiles for Submarine Power Cables," IEEE Trans. Power Del., vol. 34, no. 3, pp. 1079-086, 2019.

[10] M. Tonani, P. Sykes, R. R. King, N. McConnell, A-C. Péquignet, E. O'Dea, J. A. Graham, J. Polton and J. Siddorn, "The impact of a new high-resolution model on the Met Office North-West European Shelf Forecasting system," Ocean Sci., vol. 15, pp. 1133-1158, 2019.

[11] Electric Cables - Calculation of the current rating, Part 1-1: Current rating equations (100\% load factor) and calculation of losses General, Ed. 2.0, IEC standard 60287-1-1, 2006.

[12] T. Worzyk, Submarine Power Cables, Springer, 2009.

[13] Electric Cables - Calculation of the current rating, Part 2-1: Thermal Resistance, Ed. 2.0, IEC standard 60287-2-1, 2015.

[14] K. F. Goddard, J. A. Pilgrim, R. D. Chippendale and P. L. Lewin, "Induced Losses in Three-core SL-type High Voltage Cables," IEEE Trans. Power Del., vol. 30, no. 3, pp. 1505-1513, 2015.

[15] R. Benato, S. Dambone Sessa and M. Forzan, "Experimental Validation of Three-Dimension Multiconductor Cell Analysis by a 30 km Submarine Three-Core Armoured Cable," IEEE Trans. Power Del., vol. 33, no. 6, pp. 2910-2919, 2018. 
[16] K. G. Nayar, M. H. Sharqawy, L. D. Banchik and J. H. Lienhard V, "Thermophysical properties of seawater: A review and new correlations that include pressure dependence," Desalination, vol. 390, pp. 1-24, 2016.

[17] UNESCO, "International Oceanographic Tables Volume 4," UNESCO technical papers in marine science, vol. 40, p. 193, 1987.

[18] S. Goto and O. Matsubayashi, "Inversion of needle-probe data for sediment thermal properties of the eastern flank of the Juan de Fuca Ridge," J. Geophys. Res. Solid Earth, vol. 113, no. B8, 2008.

[19] Comsol Multiphysics Version 5.3 Reference Manual, COMSOL, 2017.

[20] Cigré, "Calculation of temperatures in ventilated cable tunnels," Electra, vol. 143, p. 38-59, 1992.

[21] D. Chatzipetros and J. A. Pilgrim, "Review of the accuracy of Single Core Equivalent Thermal Model (SCETM) for Offshore Wind Farm (OWF) cables," IEEE Trans. Power Del., vol. 33, no. 4, pp. 19131921, 2018.

[22] S. Catmull, R. D. Chippendale, J. A. Pilgrim, G. Hutton and P. Cangy, "Cyclic Load Profiles for Offshore Wind Farm Cable Rating," IEEE Trans. Power Del., vol. 31, no. 3, pp. 1242-1250, 2016.

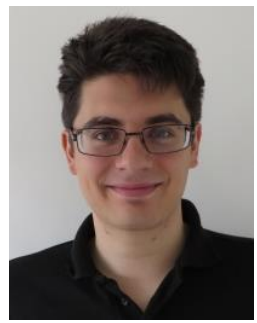

\section{BIOGRAPHIES}

George Callender was born in Basildon, UK in 1991. He received M.Sci (Hons) in Natural Sciences (Maths and Physics) from the University of Durham, UK in 2013. He received a Ph.D. degree in electrical engineering from the University of Southampton, UK in 2018. He is currently a Research Fellow in High Voltage Numerical Modelling at the University of Southampton. His research interests include partial discharge phenomena and the thermal modelling of high voltage plant.

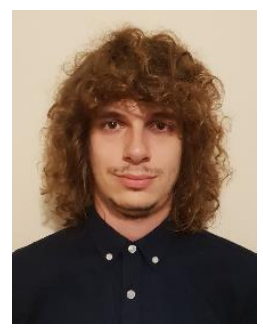

Daniel Ellis was born in Portsmouth, UK in 1996. He received his MSci in Geophysics from the University of Southampton, UK in 2018. He is currently a Research Assistant at the University of Southampton, focusing on the numerical modelling of submarine $\mathrm{HV}$ cables, working primarily with Professor Justin Dix. His research interests include marine geophysics and geology, and submarine $\mathrm{HV}$ cables.

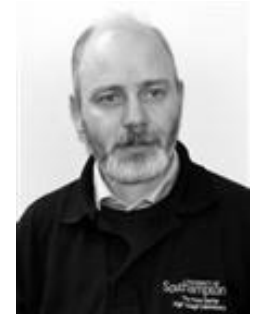

Kevin F. Goddard received a BSc in Electrical Engineering from University of Southampton, England in 1982. He obtained his PhD in 1992 for work on stray fields in the stator frames of electrical machines, also from University of Southampton.

After short periods in industry, he became a Research Fellow at University of Southampton. He worked on electromagnetic design and numerical modelling of electrical machines. His recent work involved electromagnetic and thermal modelling in cables. Dr. Goddard is a member of the IET.

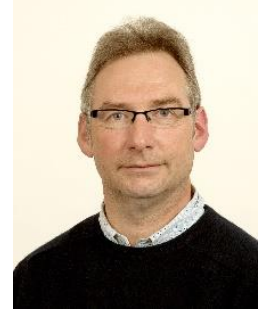

Justin K. Dix is Head of the Geology and Geophysics Research Group at the University of Southampton, Southampton, U.K. His major interests are the use of high-resolution geophysical and geological techniques to investigate the quaternary history of and modern sedimentary process operating on the continental shelf.

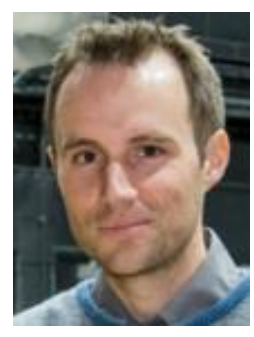

James A. Pilgrim (M'09, SM'17): Received the Bachelor's degree in electrical engineering from the School of Electronics and Computer Science at the University of Southampton in2007. He joined the staff of the University of Southampton in 2007 as a Research Assistant, gaining his PhD in2011. He joined the academic staff of the University in 2012 . His research interests include all aspects of high voltage cables and associated insulation systems. $\mathrm{He}$ is currently the Chair of the DEIS Technical Committee on Smart Grid. He is actively involved in the development of current rating methodologies, acting as the UK member of IEC TC 20 WG19 (Current Rating and Short Circuit Limits of Cables) and Cigre Working Groups B1.35 "Guide to rating calculations", B1.56 "Current rating verification" and B1.64 "Evaluation of losses in armoured three core cables".

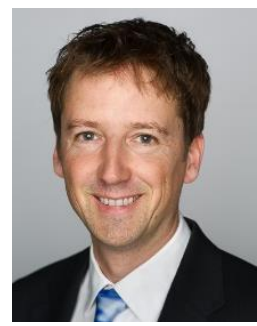

Matthias Erdmann graduated in Physics from University of Göttingen, Germany (PhD 2007) and has since then been working in several innovative energy industry companies. From 2007-2012 he was employed in photovoltaics industry focusing on the development of high-efficiency solar cells. From 2012-2016 he worked in the area of energy storage and conversion, developing software for analysis and optimization of complex energy systems and leading business case and feasibility studies for advanced renewable energy projects. In 2016 he joined AP Sensing and is now responsible for AP Sensing's power cable monitoring software solutions. 